\title{
L-Ornithine L-Aspartate (LOLA) as a Novel Approach for Therapy of Non-alcoholic Fatty Liver Disease
}

\author{
Ali Canbay ${ }^{1} \cdot$ Jan-Peter Sowa ${ }^{1}$
}

Published online: 31 January 2019

(c) The Author(s) 2019

\begin{abstract}
L-ornithine L-aspartate (LOLA) has been known as an effective ammonia-lowering agent for more than 50 years with good evidence in hepatic encephalopathy. Administration of LOLA removes ammonia via two distinct mechanisms: by synthesis of urea and by the synthesis of glutamine via the enzyme glutamine synthetase. While LOLA has been used in cirrhosis and acute liver injury settings, it is less clear if LOLA could be used in non-alcoholic fatty liver disease (NAFLD). NAFLD and the progressive form non-alcoholic steatohepatitis (NASH) are currently the leading causes of chronic liver disease worldwide, with roughly $25 \%$ of the world population affected by NAFLD. Consequences of NASH are end-stage liver disease and cardiovascular morbidity and mortality. As the basis for NAFLD is excess calorie uptake and excess adipose tissue mass, the conservative therapeutic approach is weight loss by intense lifestyle change. However, no pharmacological treatment options are currently approved. LOLA is being investigated as a pharmacological tool to ameliorate liver injury in NAFLD on the basis that it lowers liver ammonia concentrations and supplies anti-oxidative glutamine and glutathione. Indirect hepatoprotective effects currently under investigation could also be beneficial.
\end{abstract}

\section{NAFLD: Definitions, Causes and Prevalence}

Non-alcoholic fatty liver disease (NAFLD) is a spectrum of conditions based on steatosis of the liver without significant alcohol consumption ( $\geq 20 \mathrm{~g} /$ day women; $\geq 30 \mathrm{~g} /$ day men) [1]. Non-alcoholic fatty liver (NAFL) is the mere accumulation of fat as lipid droplets in hepatocytes, with non-alcoholic steatohepatitis (NASH) as progressive form with lobular inflammation and ballooning degeneration of hepatocytes. NAFLD and NASH are now recognized as liver manifestations of the metabolic syndrome and as such based on excess calorie intake, insufficient activity and exercise, and the resultant excess adipose tissue depots. Risk factors for NAFLD include obesity, diabetes mellitus type 2 (T2DM), hypertension, and dyslipidemia with or without elevated liver enzymes. There are other possible causes for liver steatosis, i.e. HCV infection, drugs and genetic causes (celiac disease, Wilson's disease, lysosomal acid

Ali Canbay

Ali.canbay@med.ovgu.de

1 Department of Gastroenterology, Hepatology, and Infectious Diseases, Otto-von-Guericke University Magdeburg,

Leipziger Str. 44, 39120 Magdeburg, Germany lipase deficiency), which should be excluded upon diagnosis. However, as the global prevalence of NAFLD has risen to approximately $25 \%$ following the obesity pandemic [2], most cases of NAFLD and NASH are due to overweight and obesity.

\section{Pathophysiology of NAFLD and NASH}

Despite the simple basis for NAFLD, which is excess adipose tissue mass with subsequently altered release patterns for fatty acids and adipokines, the actual pathophysiology of NAFLD is highly complex. Excess supply of triglyceride and glucose leads to increased de novo lipogenesis and altered formation of lipoprotein particles [3]. Oxidative stress and endoplasmic reticulum stress due to reduced mitochondrial fatty acid oxidation and increased peroxisomal fatty acid oxidation lead to cellular injury, cell death of hepatocytes and subsequently inflammation [4]. Liver injury from this variety of processes is summarized in the "multiple parallel hits theory", as it seems that the combination of many factors promotes the progression from NAFLD to NASH [5]. It is important to note, that there may be different types of NAFLD [6]. One type is based solely on the metabolic insult, with significant contribution of hepatic insulin 
resistance to disease progression and mostly cardiovascular morbidity and mortality. The other type is genetically predetermined injury (i.e. PNPLA3 variants), with more prominent injury and inflammation of the liver and higher risk of progression to cirrhosis or hepatocellular carcinoma (HCC) [6]. However, this theory needs to be investigated in appropriate settings. Accumulating data suggests that NAFLD is not a bystander of the metabolic syndrome but the cause of progression of insulin resistance and adverse cardiovascular outcomes [7, 8]. In particular elevated de novo lipogenesis and gluconeogenesis in hepatocytes under surplus of lipids and glucose indicates deranged hepatic metabolism as a central component for NAFLD development. Since the liver is the main metabolic organ responsible for lipid and glucose metabolism, liver function is essential for all organs, as it includes allocation of transport proteins, nutrients, and energy supply to the whole organism. In that sense, NAFLD comprises a systemic disease with multiple interactions with other organs.

\section{Current Conservative and Pharmacological Treatments of NAFLD}

The underlying cause for NAFLD is excess lipid accumulation within the hepatocytes. However, the exact mechanisms of NAFLD development and progression to NASH are incompletely understood and involve complex interacting metabolic pathways and processes (as described above in 2). The only currently available conservative treatment option, is to counter the underlying overweight and obesity by weight reduction, which will positively affect all co-morbidities including insulin resistance [1]. A relevant effect on NAFLD can only be achieved by weight loss of approximately $10 \%$ of the initial weight, which requires intense life-style changes. It is needless to say, that many patients do not reach this target or cannot maintain it permanently. Weight loss can also be achieved by bariatric surgery, which is generally effective but also comes with risks of large abdominal surgeries. Up to now, no pharmacological treatment targeting NAFLD has been approved, although there are some promising candidate substances in clinical trials (Table 1). In the USA, treatment with combinations of Vitamin E and pioglitazone or liraglutide is commonly applied, but this is not approved for Europe. Detailed recommendations on treatment of NAFLD can be found in the Clinical Management Guidelines of the European Associations for the Study of the Liver (EASL), of Diabetes (EASD), and of Obesity (EASO) [1]. One of the reasons for the absence of uncontroversial effective pharmacotherapy for NAFLD is the high complexity of this disease, caused by multiple interacting factors. In addition, prior candidate substances and their targets were initially identified in murine models of NAFLD or NASH. However, there is only little overlap of concordantly regulated genes between murine models of NASH and human transcriptomes in NASH [9]. Limitations of the utilized models for NAFLD or NASH comprise the mechanisms of disease generation, the different genetic background, and anatomical differences in the digestive system between mouse and human. Thus, it is not surprising that potential targets for NASH therapy identified in murine models might not give similar results in clinical studies.

On an empirical basis, multiple possible targets for pharmacotherapy are promising: the insulin resistance of the liver and systemically, and factors affecting the liver itself. Candidate substances in Phase III clinical trials are obeticholic acid (6-ethylchenodeoxycholic acid), elafibranor, cenicriviroc, and selonsertib (Table 1). For details on the current situation on pharmacological treatment options for NASH, please refer to the review by Alkhouri et al. [10].

\section{Rationale for LOLA as Possible Treatment for NAFLD}

Among the many functions of the liver amino acid metabolism, aa catabolism and ammonia detoxification are performed in hepatocytes. These have not yet been explored as potential targets to treat NAFLD or NASH. L-ornithine L-aspartate (LOLA) is a mixture of endogenous amino acids

Table 1 Pharmacological components for treatment of NAFLD or NASH in phase III clinical trials. Table adapted from [10]

\begin{tabular}{llc}
\hline Substance/label & Target/mode of action & (Expected) effect \\
\hline Obeticholic acid & Agonist of farnesoid-X-receptor & $\begin{array}{c}\text { Improvement of insulin sensitivity and reduced gluconeogen- } \\
\text { esis and serum triglycerides }\end{array}$ \\
Elafibranor & $\begin{array}{l}\text { Dual agonist for peroxisome proliferator-activated receptor- } \alpha \\
\text { and }-\delta\end{array}$ & $\begin{array}{c}\text { Improvement of glucose homeostasis and insulin metabolism } \\
\text { and reduction of inflammation. }\end{array}$ \\
Cenicriviroc & Chemokine (C-C motif) receptor types 2 and 5 antagonist & $\begin{array}{c}\text { Reduced inflammatory response to hepatocyte injury by } \\
\text { Kupffer cells, subsequently diminished fibrosis }\end{array}$ \\
Selonsertib & Apoptosis signal-regulating kinase 1 inhibitor & $\begin{array}{c}\text { Reduction of hepatic inflammation, hepatocyte apoptosis and } \\
\text { fibrosis due to oxidative stress }\end{array}$
\end{tabular}


supposed to facilitate ammonia removal in patients with liver cirrhosis [11]. In the following section we suggest a hypothetical approach to treat metabolic alterations in NAFLD with LOLA. Some of the potential effects are currently under investigation and thus are only speculative.

\subsection{Known Hepatoprotective Effects of LOLA}

Nitrogen metabolism in muscle and other peripheral tissues leads to formation of ammonia, which is converted via urea cycle in the liver and excreted as urine [12]. Impaired liver function results in elevation of ammonia concentration [13]. In acute, acute-on-chronic and chronic liver failure, ammonia accumulates through diminished activity of the urea cycle. High serum concentrations of ammonia are associated with hepatic encephalopathy (HE), hepatocyte apoptosis, inflammation, liver function impairment, and high mortality $[13,14]$.

LOLA is used in patients with liver cirrhosis to ameliorate minimal HE by enhancing ammonia removal in still functional hepatocytes [15]. It is supposed that LOLA affects ammonia removal on the one hand by increased synthesis of urea and on the other hand by increased synthesis of glutamine via the enzyme glutamine synthetase (GS) [15]. In addition, LOLA-derived glutamate can also be converted to glutathione, which is an important anti-oxidant for hepatocytes. In addition to direct effects of LOLA on the liver due to enhanced ammonia removal and improved antioxidative capacity, there might be indirect hepatoprotective effects due to partial reversal of sarcopenia [16] and due to nitric oxide (NO) formation from L-arginine, resulting in improved hepatic microcirculation [17]. These putative direct and indirect effects of LOLA have been demonstrated in pre-clinical and small clinical studies, awaiting confirmation in larger trials (Fig. 1).

\subsection{Ammonia as Possible Contributor to Liver Injury in NAFLD}

Apart from the known elevations of circulating ammonia in cirrhosis and acute liver failure, one study investigated ammonia content of the liver and HE in chronic liver diseases, including NAFLD [15]. Given the key role of the liver for the removal of excess ammonia, it is not surprising that patients with chronic liver diseases are hyperammonemic [18] and that hepatic accumulation of ammonia has been found in both patients and animal models of NAFLD or NASH [12]. Histological staining of liver tissue for ammonia content seems to correlate well with disease severity of NAFLD with higher concentrations in NASH [12]. These data imply that in NAFLD and in particular in progressive $\mathrm{NASH}$, ammonia accumulates in the liver. The functional background of this might be reduced expression of urea cycle enzymes by fatty acid accumulation [19]. Another possible mechanism might be reduced flux through the GS pathway by up to $50 \%$, as demonstrated in liver biopsy samples from patients with histologically proven steatosis and raised serum transaminases [20]. Ammonia has thus been suggested as possible target for NAFLD treatment [14]. In a recent study, hypermethylation of urea cycle gene as well as reduced expression and activity of urea a cycle enzymes have been demonstrated in human NASH and a rat model of NASH [21]. This was associated with elevated plasma ammonia concentrations and ammonia accumulation in liver tissue of patients with NASH.

\subsection{Efficacy of LOLA in Pre-clinical Models and Studies of NAFLD}

Until now, most studies on the efficacy of LOLA have been performed in cirrhosis or models thereof. However, there are a few preclinical studies which could indicate a possible effect of LOLA on NAFLD. Two studies in experimental cirrhosis and HE described that ammonia lowering improved sarcopenia in cirrhotic Sprague-Dawley rats by stabilizing amino acid metabolism [22], and that LOLA treatment resulted in a significant increase of plasma glutamine, which probably derived from L-ornithine via GS [23]. Glutamine has been shown to be protective in preclinical models of NAFLD and NASH, possibly by reducing oxidative stress $[24,25]$. Indirect evidence suggests that LOLA could diminish liver injury in NAFLD by reducing ammonia and oxidative stress.

More direct evidence for a possible use of LOLA as a treatment for NAFLD comes from early clinical studies. Almost two decades ago, it was shown that LOLA could attenuate serum AST, ALT and $\gamma$-GT levels by up to $70 \%$ in chronic liver diseases. This effect was not only dose related but was more pronounced in patients with fatty liver compared to patients with cirrhosis [26]. In a more recent study, a Chinese group performed a multicenter openlabel, multidose, randomized controlled trial comprising 72 patients to assess the efficacy of orally administered LOLA for 12 weeks. The treatment resulted in a significant doserelated reduction in ALT and triglycerides. Liver/spleen ratios in computed tomography examination improved significantly after LOLA treatment [27]. However, serum transaminase levels are strongly limited to assess liver injury specifically for NAFLD. Further studies with more reliable outcome markers are needed to verify possible effects of LOLA for NAFLD treatment. In a very recent trial with 78 patients with NASH, administration of LOLA seemed to improve hepatic microcirculation in the presence of mild fibrosis, determined by transient elastography [28]. In these clinical studies LOLA appears to reduce liver injury in NAFLD patients. These findings are limited by the use of 
(a)

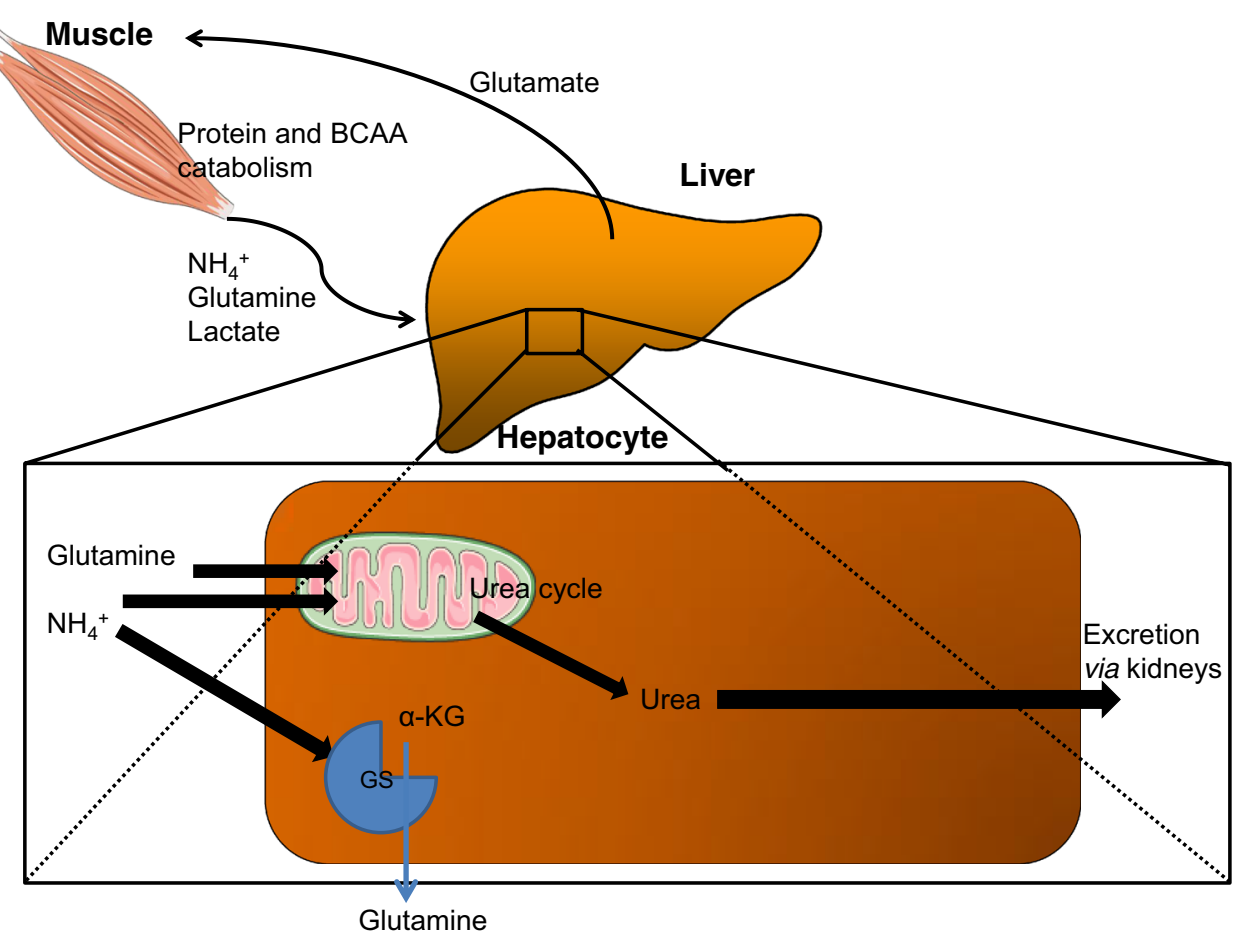

(b)

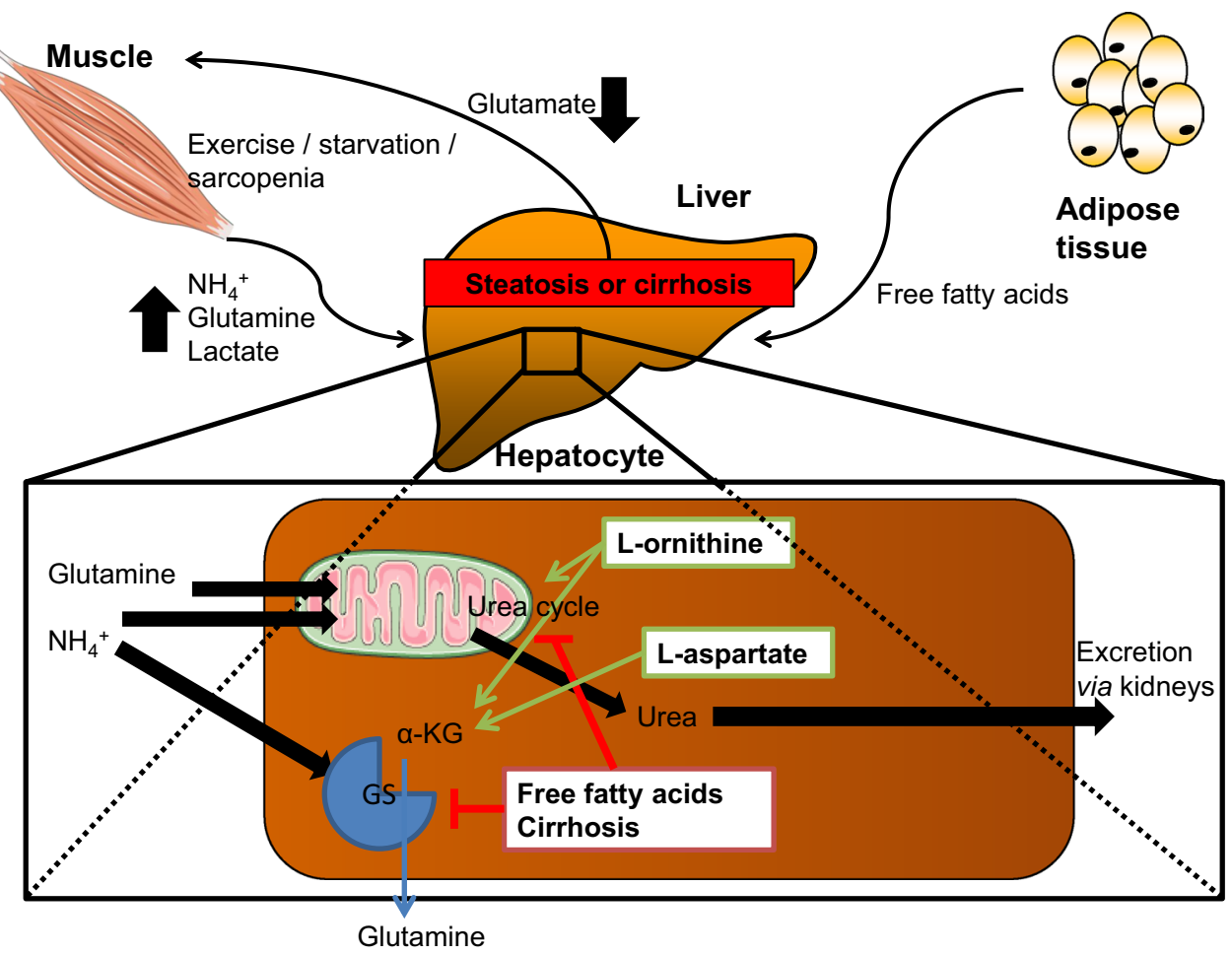

serum transaminases as main outcome measure, which are not reliable markers to asses NAFLD or NASH. Larger studies in more diverse populations are required, employing liver fat content and liver inflammation as indicators for efficacy, to substantiate these initial findings.

\section{Summary and Conclusions}

Current data support the crucial role of ammonia in advanced liver diseases. Data from preclinical models and from a small number of clinical trials support the thesis that LOLA has hepatoprotective properties in cirrhosis. In 
४Fig. 1 Ammonia generation and detoxification and putative mechanistic targets of LOLA for treatment of NAFLD. a Ammonia detoxification under healthy conditions: ammonia $\left(\mathrm{NH}_{3}\right)$ is generated as byproduct from protein and branched chain amino acid (BCAA) catabolism and transported systemically as ammonium $\left(\mathrm{NH}_{4}{ }^{+}\right)$. Detoxification of $\mathrm{NH}_{4}^{+}$in the liver is performed either via the urea cycle, part of which is located within mitochondria, or by the enzyme glutamine synthetase (GS). For these reactions, glutamine and $\alpha$-ketoglutarate $(\alpha-\mathrm{KG}$; derived from BCAA or malate) are required. b Supposed effects of steatosis or cirrhosis on ammonia detoxification (red) and putative mechanism of L-ornithine L-aspartate (LOLA; green). In cirrhosis, performance of urea cycle is diminished and glutamine as well as $\alpha-\mathrm{KG}$ reserves are depleted, leading to $\mathrm{NH}_{4}{ }^{+}$accumulation and toxic effects, i.e. hepatic encephalopathy. LOLA is supposed to enhance urea cycle via L-ornithine and L-ornithine-derived glutamate. Both L-ornithine and L-aspartate can replenish $\alpha-\mathrm{KG}$ reserves. In addition, L-ornithine might be able to supply anti-oxidative capacity by restoring glutathione and L-aspartate-derived nitric oxide might improve hepatic microcirculation (both not shown)

patients with NAFLD or NASH, LOLA appears to reduce serum concentrations of liver enzymes and triglycerides. Putative mechanisms for the effect of LOLA in NAFLD are enhanced ammonia removal, increased anti-oxidative capacity, and attenuated lipid peroxidation by glutamine and glutathione, improved hepatic microcirculation due to L-arginine-derived NO. These effects should be investigated in well-controlled clinical settings to demonstrate if LOLA could be a feasible treatment for NAFLD or NASH.

\section{Compliance with Ethical Standards}

Funding This article is published in a special edition journal supplement wholly funded by Merz Pharmaceuticals GmbH, Frankfurt, Germany.

Conflict of interest Prof. Ali Canbay has received a research grant from MERZ and received speaker honoraria from MERZ, Falk, Shire, and GILEAD. Jan-Peter Sowa declares that he has no conflict of interest.

Open Access This article is distributed under the terms of the Creative Commons Attribution-NonCommercial 4.0 International License (http://creativecommons.org/licenses/by-nc/4.0/), which permits any noncommercial use, distribution, and reproduction in any medium, provided you give appropriate credit to the original author(s) and the source, provide a link to the Creative Commons license, and indicate if changes were made.

\section{References}

1. European Association for the Study of the Liver (EASL), European Association for the Study of Diabetes (EASD), European Association for the Study of Obesity (EASO). EASL-EASDEASO Clinical Practice Guidelines for the management of nonalcoholic fatty liver disease. J Hepatol. 2016;64:1388-402.

2. Younossi ZM, Koenig AB, Abdelatif D, Fazel Y, Henry L, Wymer M. Global epidemiology of nonalcoholic fatty liver
disease-Meta-analytic assessment of prevalence, incidence, and outcomes. Hepatol Baltim Md. 2016;64:73-84.

3. Vernon G, Baranova A, Younossi ZM. Systematic review: the epidemiology and natural history of non-alcoholic fatty liver disease and non-alcoholic steatohepatitis in adults. Aliment Pharmacol Ther. 2011;34:274-85.

4. Angulo P. Nonalcoholic fatty liver disease. N Engl J Med. 2002;346:1221-31.

5. Tilg H, Moschen AR. Evolution of inflammation in nonalcoholic fatty liver disease: the multiple parallel hits hypothesis. Hepatol Baltim Md. 2010;52:1836-46.

6. Gastaldelli A. Insulin resistance and reduced metabolic flexibility: cause or consequence of NAFLD? Clin Sci Lond Engl. 1979;2017(131):2701-4.

7. Fabbrini E, Magkos F, Mohammed BS, Pietka T, Abumrad NA, Patterson BW, et al. Intrahepatic fat, not visceral fat, is linked with metabolic complications of obesity. Proc Natl Acad Sci USA. 2009;106:15430-5.

8. Vanni E, Bugianesi E, Kotronen A, De Minicis S, Yki-Järvinen H, Svegliati-Baroni G. From the metabolic syndrome to NAFLD or vice versa? Dig Liver Dis Off J Ital Soc Gastroenterol Ital Assoc Study Liver 2010;42:320-30.

9. Teufel A, Itzel T, Erhart W, Brosch M, Wang XY, Kim YO, et al. Comparison of gene expression patterns between mouse models of nonalcoholic fatty liver disease and liver tissues from patients. Gastroenterology. 2016;151(513-525):e0.

10. Alkhouri N, Scott A. An update on the pharmacological treatment of nonalcoholic fatty liver disease: beyond lifestyle modifications. Clin Liver Dis. 2018;11:82-6.

11. Butterworth RF, Kircheis G, Hilger N, McPhail MJW. Efficacy of 1-ornithine 1-aspartate for the treatment of hepatic encephalopathy and hyperammonemia in cirrhosis: systematic review and meta-analysis of randomized controlled trials. J Clin Exp Hepatol. 2018;8(3):301-13.

12. Gutiérrez-de-Juan V, López de Davalillo S, Fernández-Ramos D, Barbier-Torres L, Zubiete-Franco I, Fernández-Tussy P, et al. A morphological method for ammonia detection in liver. PLoS One. 2017; 12:e0173914.

13. Jepsen P, Ott P, Andersen PK, Sørensen HT, Vilstrup H. Clinical course of alcoholic liver cirrhosis: a Danish population-based cohort study. Hepatol Baltim Md. 2010;51:1675-82.

14. Thomsen KL, De Chiara F, Rombouts K, Vilstrup H, Andreola F, Mookerjee RP, et al. Ammonia: a novel target for the treatment of non-alcoholic steatohepatitis. Med Hypotheses. 2018;113:91-7.

15. Butterworth RF, Canbay A. Hepatoprotection by L-ornithine L-aspartate in non-alcoholic fatty liver disease. Dig Dis Basel Switz. 2018;17:1-6.

16. Pasha Y, Leech R, Violante IR, Cook N, Crossey MME, TaylorRobinson SD. The brain-muscle axis in minimal hepatic encephalopathy (MHE): a placebo-controlled, longitudinal double-blind trial with L-Ornithine 1-Aspartate (LOLA) - preliminary results. J Clin Exp Hepatol. 2017;7:S5-6.

17. Kus K, Walczak M, Maslak E, Zakrzewska A, Gonciarz-Dytman A, Zabielski P, et al. Hepatoselective nitric oxide (NO) donors, V-PYRRO/NO and V-PROLI/NO, in nonalcoholic fatty liver disease: a comparison of antisteatotic effects with the biotransformation and pharmacokinetics. Drug Metab Dispos Biol Fate Chem. 2015;43:1028-36.

18. Felipo V, Urios A, Montesinos E, Molina I, Garcia-Torres ML, Civera $\mathrm{M}$, et al. Contribution of hyperammonemia and inflammatory factors to cognitive impairment in minimal hepatic encephalopathy. Metab Brain Dis. 2012;27:51-8.

19. Tomomura M, Tomomura A, Dewan MA, Saheki T. Long-chain fatty acids suppress the induction of urea cycle enzyme genes by glucocorticoid action. FEBS Lett. 1996;399:310-2. 
20. Kaiser S, Gerok W, Häussinger D. Ammonia and glutamine metabolism in human liver slices: new aspects on the pathogenesis of hyperammonaemia in chronic liver disease. Eur J Clin Invest. 1988; 18:535-42.

21. De Chiara F, Heebøll S, Marrone G, Montoliu C, Hamilton-Dutoit $\mathrm{S}$, Ferrandez A, et al. Urea cycle dysregulation in non-alcoholic fatty liver disease. J Hepatol. 2018;69:905-15.

22. Kumar A, Davuluri G, Silva RNE, Engelen MPKJ, Ten Have GAM, Prayson R, et al. Ammonia lowering reverses sarcopenia of cirrhosis by restoring skeletal muscle proteostasis. Hepatol Baltim Md. 2017;65:2045-58.

23. Rose C, Michalak A, Pannunzio P, Therrien G, Quack G, Kircheis $\mathrm{G}$, et al. L-ornithine-L-aspartate in experimental portal-systemic encephalopathy: therapeutic efficacy and mechanism of action. Metab Brain Dis. 1998;13:147-57.

24. Lin Z, Cai F, Lin N, Ye J, Zheng Q, Ding G. Effects of glutamine on oxidative stress and nuclear factor- $\mathrm{\kappa B}$ expression in the livers of rats with nonalcoholic fatty liver disease. Exp Ther Med. 2014;7:365-70.

25. Sellmann C, Jin CJ, Degen C, De Bandt J-P, Bergheim I. Oral glutamine supplementation protects female mice from nonalcoholic steatohepatitis. J Nutr. 2015;145:2280-6.

26. Grüngreiff K, Lambert-Baumann J. Efficacy of L-ornithin-L-aspartate-granules in chronic liver diseases. Medizinische Weltstuttgart. 2001;219:p.

27. Tian L, Lu L, Tang C, Xie Y, Luo H, Tan S, et al. [Aspartateornithine granules in the treatment of nonalcoholic steatohepatitis: a multiple-dose parallel controlled clinical trial]. Zhonghua Gan Zang Bing Za Zhi Zhonghua Ganzangbing Zazhi Chin. J Hepatol. 2013;21:528-32.

28. Ermolova T, Ermolov S. Correction of intrahepatic microcirculation disorders by L-ornithine-L-aspartate at the chronic liver diseases patients. J Hepatol. 2018;68:S585-6 (abstract). 